\title{
Problemas y aciertos de la teoría del Yo narrativo de Dennett: aportaciones al debate sobre la identidad personal 1
}

\section{Weaknesses and Strengths of Dennett's Narrative Self Theory: Contributions to the Debate on Personal Identity}

\author{
Alfonso MuÑoz CORCUERA \\ (Universidad Complutense de Madrid)
}

Recibido: 06/11/2012

Aceptado: 19/03/2013

\section{Resumen}

En este artículo se hace un análisis crítico de la teoría del Yo narrativo de Dennett en tanto teoría de la identidad personal. Se contextualiza su aparición en el debate sobre la identidad personal en la década de 1980, se presenta su teoría a la luz del análisis que hizo Strawson de la Narratividad, se ponen en relación las críticas recibidas por la teoría de Dennett y por el enfoque narrativo en su conjunto y finalmente se muestra cómo la teoría de Dennett podría ayudar a los actuales defensores de las teorías narrativas de la identidad a reformular sus argumentos en respuesta a las críticas recibidas. Se espera así contribuir por un lado a una mejor comprensión de la teoría de Dennett y por otro reivindicar su vigencia en la situación actual del debate sobre la identidad personal en la filosofía analítica.

Palabras clave: identidad personal, yo, Narratividad, Dennett, Strawson.

\section{Abstract}

This article presents a critical assesment of Dennett's narrative self theory as a personal identity theory. First it is contextualized in regard to the debate on person-

\footnotetext{
1 Este artículo ha sido realizado en el marco del proyecto «Normatividad y praxis. El debate actual después de Wittgenstein», del Ministerio de Ciencia e Innovación (FFI2010-15975).
} 
al identity in the decade of 1980, then Dennett's theory is presented in the light of Strawson's analysis of Narrativity, the criticisms received by Dennett's theory and by narrative theories in general are put in relationship, and finally it is shown how Dennett's theory could help the actual supporters of narrative identity theories to reformulate their arguments in defense to the received criticisms. Thus I hope to contribute to a better understanding of Dennett's theory and to claim for its relevance in the actual debate about personal identity in analytic philosophy.

Keywords: Personal identity, Self, Narrativity, Dennett, Strawson.

\section{Introducción al problema de la identidad personal}

El problema de la identidad personal tal y como es abordado hoy en día en la filosofía analítica tiene sus orígenes a mediados del siglo XX cuando autores como Williams, Shoemaker, Lewis, Parfit o Perry 2 recogieron el planteamiento hecho por Locke en el siglo XVII. Para Locke la identidad de las personas debía definirse en términos de la continuidad de la conciencia, tomando la memoria como el elemento capaz de unificar ésta a través de las interrupciones cotidianas que sufre a causa del sueño o de pequeños periodos de olvido. ${ }^{3}$ La teoría de Locke fue entendida como una teoría simple basada en la continuidad de la memoria, lo que tuvo dos consecuencias. La primera, es que se reveló como patentemente falsa ya desde el siglo XVIII, recibiendo duras críticas que no fueron respondidas en siglos. ${ }^{4} \mathrm{La}$ segunda, es que el criterio de continuidad de la memoria fue entendido por los lectores de Locke en el siglo XX como un criterio objetivo que se podía contraponer a otro criterio objetivo: el de la continuidad corporal. Así, el debate que se dio desde mediados del siglo XX hasta aproximadamente la década de 1980 estuvo dominado por los intentos de elaborar un enfoque objetivo del problema de la identidad personal en el que los partidarios de los criterios físicos -Williams- se opusieron a los partidarios de los criterios psicológicos -Shoemaker, Lewis, Parfit, Perry-.

\footnotetext{
2 Williams, B.: "Personal Identity and Individuation", Proceedings of the Aristotelian Society, 57, 1957, pp. 229-252; Shoemaker, S.: "Personal Identity and Memory", The Journal of Philosophy, 56, 1959, pp. 868-882; Lewis, D. K.: Supervivencia e identidad, Cuadernos de crítica, 27, México, Universidad Nacional Autónoma de México, 1976/1984; Parfit, D.: Identidad personal, Cuadernos de crítica, 25, México, Universidad Nacional Autónoma de México, 1971/1984; Perry, J.: "Can the Self Divide?", The Journal of Philosophy, 69, 1972, pp. 463-488.

3 Locke, J.: Ensayo sobre el entendimiento humano, México, Fondo de Cultura Económica, 1690/1999, pp. 318-319.

4 Butler, J.: "Of Personal Identity", en J. Perry (ed.), Personal Identity, Berkeley and Los Angeles, University of California Press, 2008; Reid, T.: "Of Mr. Locke's Account of Our Personal Identity", en J. Perry (ed.), Personal Identity, op. cit.
} 
Sin duda los partidarios de los criterios psicológicos, que reformularon con éxito la teoría de Locke para dar respuesta a la mayoría de las críticas que había recibido, representaron la postura dominante en dicho debate. Para ellos los «Yoes» o las «personas»-términos sinónimos en este contexto- eran una entidad mental definida en términos de la continuidad psicológica (a la memoria unieron la continuidad de otras conexiones psicológicas como los deseos, las creencias o las intenciones) que debía diferenciarse de los seres humanos que también somos. El motivo para sostener esto era que, aunque normalmente la continuidad física y la continuidad psicológica coinciden, si imaginamos un caso hipotético en el que ambas divergiesen -duplicaciones, teletransportaciones, trasplantes cerebrales...- algunas de la características de las personas a las que concedemos gran importancia parecerían seguir a la continuidad psicológica y no a la física o biológica.

Schechtman señala que el problema de la identidad personal para los partidarios del enfoque objetivo se vinculaba fundamentalmente a cuatro características específicas de las personas: la responsabilidad moral, la preocupación prudencial, la compensación y, sobre todo, la supervivencia. ${ }^{5}$ En este sentido la pregunta que se puede considerar central en el debate era acerca de las condiciones de supervivencia de las personas, o más exactamente, acerca de qué es lo que nos importa que se mantenga en una determinada situación para decir que hemos sobrevivido. Sin embargo, como Parfit puso de manifiesto en Razones y personas, si adoptamos un enfoque objetivo al final nos vemos forzados a admitir que lo que nos importa -la continuidad psicológica- no está necesariamente relacionado con la identidad personal. Es decir, que lo que nos importa (o debería importar) en nuestra supervivencia no es que seamos nosotros los que sobrevivamos, sino que sea alguien que se parezca lo suficiente a nosotros. Por decirlo en palabras de Parfit, la identidad personal no es lo que importa. 6

La extravagancia de esta afirmación -que Parfit venía repitiendo desde tiempo atrás-, y sobre todo su inapelabilidad, hizo que, a partir de los años ochenta, un nuevo enfoque empezase a abrirse camino en el debate sobre la identidad personal dejando a un lado el enfoque objetivo. Se trata de las teorías narrativas, entre las que podemos destacar las de MacIntyre, Bruner, Taylor, Ricoeur, Schechtman ${ }^{7}$ y la del autor que nos ocupará en las próximas páginas, Dennett. Estas teorías se puede interpretar que tratan de reformular la continuidad psicológica del enfoque objetivo en términos de la continuidad de la narración que contamos sobre nosotros mis-

\footnotetext{
5 Schechtman, M.: The Constitution of Selves, Ithaca, NY, Cornell University Press, 1996, pp. 136162.

6 Parfit, D.: Razones y personas, Boadilla del Monte (Madrid), A. Machado Libros, 1984/2004, p. 458.

7 MacIntyre, A.: Tras la virtud, Barcelona, Crítica, 1981/2004; Bruner, J.: "Life as Narrative", Social Research, 54, 1987, pp. 11-32; Taylor, C.: Fuentes del yo: La construcción de la identidad moderna, Barcelona, Paidós, 1989/1996; Ricoeur, P.: Sí mismo como otro, Madrid: Siglo XXI, 1990/1996; Schechtman, The Constitution of Selves. op. cit.
} 
mos, ${ }^{8}$ y si bien su variedad es enorme, siguiendo a Strawson podemos decir que tienen por lo general dos tesis en común. La primera es la tesis psicológica de la Narratividad, que dice que las personas experimentan subjetivamente su vida en forma narrativa. La segunda es la tesis ética de la Narratividad, que señala que comprender la propia vida en forma narrativa es algo necesario para tener una vida buena o para desarrollarse plenamente como persona. ${ }^{9}$ Es importante señalar que no todas las teorías narrativas aceptan ambas tesis tal y como las formula Strawson, pues sin ir más lejos la teoría de Dennett no es tan explícita en el tema de la ética. Sin embargo, como veremos más adelante, si interpretamos esta segunda tesis de forma más laxa y la relacionamos no con el tener una vida buena sino con la capacidad para integrarse con normalidad en la vida social atendiendo a las cuatro características señaladas por Schechtman también la teoría de Dennett participa de ella.

En cualquier caso, a pesar del entusiasmo inicial, en los últimos años tanto la teoría de Dennett en particular como el enfoque narrativo en general han sido acusados de ser radicalmente erróneos por diversos autores. ${ }^{10}$ No creo que los argumentos que se han esgrimido en su contra sean definitivos, pero sí que han tenido un impacto enorme entre los actuales defensores del enfoque narrativo, que aún no han acertado a responder con contundencia a todas las objeciones presentadas. Entre estos últimos ya no se encuentra Dennett, quien hace años que no se ocupa de estos problemas. No obstante creo que algunos aspectos de su teoría podrían servir de ayuda a la hora de reformular el enfoque narrativo, motivo por el cual me gustaría volver a llamar la atención sobre ella.

En las próximas páginas expondré primero las características de la teoría del Yo como centro de gravedad narrativa de Dennett. A continuación señalaré las críticas que su teoría ha recibido desde su aparición, relacionándolas con las que en los últimos años ha recibido el planteamiento narrativo en general. Por último mostraré algunas de las respuestas que otros teóricos de la narratividad han esgrimido en su defensa y cómo la teoría de Dennett podría utilizarse para complementarlas.

\footnotetext{
8 Slors, M.: “Two Conceptions of Psychological Continuity", Philosophical Explorations, 1, 1998, pp. 61-80.

9 Strawson, G.: Contra la Narratividad, Cuadernos de crítica, México, Universidad Nacional Autónoma de México, 2004/en prensa.

10 Vid. Carr, D.: "Phenomenology and Fiction in Dennett", International Journal of Philosophical Studies, 6, 1998, pp. 331-344; Christman, J.: "Narrative Unity as Condition of Personhood", Metaphilosophy, 35, 2004, pp. 695-713; Lamarque, P.: "On Not Expecting Too Much From Narrative", Mind \& Language, 19, 2004, pp. 393-408; Lamarque, P.: "On the Distance between Literary Narratives and Real-Life Narratives", en D. D. Hutto (ed.), Narrative and Understanding Persons, Cambridge, Cambridge University Press, 2007, pp. 117-132; McCarthy, J.: Dennett and Ricoeur on the Narrative Self, Amherst, NY, Humanity Books, 2007; Strawson, G.: Contra la Narratividad, op. cit.; Velleman, J. D.: "The Self as Narrator", en Self to Self: Selected Essays, Cambridge, Cambridge University Press, 2006, pp. 203-223; Vice, S.: "Literature and the Narrative Self", Philosophy, 78, 2003, pp. 93-108; Zahavi, D.: "Self and Other: The Limits of Narrative Understanding", en D. D. Hutto (ed.), Narrative and Understanding Persons, op. cit., pp. 179-201.
} 


\section{La teoría del Yo de Dennett}

La primera aportación de Dennett a las teorías del Yo narrativo es una de las más tempranas, pues aunque tradicionalmente se considera que el libro Tras la virtud de MacIntyre es la obra fundacional de esta corriente, el germen de la teoría del Yo como centro de gravedad narrativa -nombre por el que se conoce la teoría de Dennett- se encuentra en el libro El ojo de la mente que Dennett editó junto a Hofstadter en el mismo año en que se publicó la obra de MacIntyre. En este peculiar libro Dennett y Hofstadter recogen textos literarios y filosóficos y los comentan desde sus posturas con respecto al problema de la conciencia. En este contexto, las primeras reflexiones de Dennett sobre el Yo narrativo surgen mientras comenta el relato de Borges «Las ruinas circulares», analizando la posibilidad de que, en última instancia, nuestros Yoes no sean más que una entidad soñada -o literariamente creada- por nuestro cuerpo. 11

Dos años después Dennett recogerá y ampliará estas reflexiones para dar lugar al texto que estas páginas pretenden acompañar, y que fue presentado originalmente en el Simposio de Houston sobre la naturaleza del Yo y la conciencia celebrado en 1983.12 Publicado en primer lugar en el Times Literary Supplement en 1988 bajo el título de «Why everyone is a novelist» y finalmente con ligeras variaciones en 1992 bajo el título de «The Self as a Center of Narrative Gravity» en el libro Self and Consciousness: Multiple Perspectives que recoge las actas del Simposio, el texto presenta las reflexiones de Dennett sobre el problema del Yo de forma mucho más desarrollada que en El ojo de la mente, por lo que es considerado por muchos la primera expresión de su teoría.

Posteriormente Dennett volvió a tratar el tema del Yo de forma más lateral en el libro La libertad de acción -en el que se ocupó de los problemas del libre albedrío y de la responsabilidad moral-, 13 y de forma específica en el artículo «The Origins of Selves», en el que trató de ilustrar la idea de que somos aquello que controlamos y por lo que nos preocupamos, lo cual se construye y modifica mediante el lenguaje y la narración que todos contamos sobre nosotros mismos. ${ }^{14}$

Todas las reflexiones presentes en estos trabajos se amalgamaron para dar forma a «La realidad de los yos», uno de los capítulos de su libro La conciencia explica$d a$. En sus páginas, que incluso reproducen literalmente algunos párrafos publica-

11 Dennett, D. C.: "Reflexiones", en D. R. Hofstadter y D. C. Dennett (eds.), El ojo de la mente: Fantasías y reflexiones sobre el yo y el alma, Buenos Aires, Editorial Sudamericana, 1981/1983, pp. 348-352.

12 Dennett, D. C.: "El Yo como centro de gravedad narrativa", Logos: Anales del Seminario de Metafisica, 1988/2013, vol. 46.

13 Dennett, D. C.: La libertad de acción: Un análisis de la exigencia de libre albedrío, Barcelona, Gedisa, 1984/2000, pp. 91-119.

14 Dennett, D. C.: "The Origins of Selves", Cogito, 3, 1989, pp. 163-173. 
dos anteriormente en los textos que acabamos de reseñar, Dennett expone la versión más desarrollada de su teoría. Sin embargo no modifica las ideas que había presentado hasta el momento, sino que por un lado las articula y por otro las sintetiza. 15 Por este motivo no tiene sentido detenerse a analizar las características específicas del Yo para Dennett en cada texto y su evolución a lo largo del tiempo -pues todos ellos conforman una unidad-, pero tampoco lo tiene quedarse con la formulación presente en este texto más completo, pues en la síntesis se pierden algunos aspectos clave para su completa comprensión. Siendo así podemos permitirnos presentar la teoría de Dennett como un todo diseminado en distintas publicaciones. 16

En cualquier caso, para poder comprender la teoría del Yo como centro de gravedad narrativa es imprescindible conocer primero aunque sea los aspectos básicos de la teoría de la conciencia de Dennett, que en La conciencia explicada bautizó como modelo de «Versiones Múltiples» y que posteriormente publicitó como modelo de la «Popularidad Cerebral».17 Este modelo establece que la conciencia, tal y como ha sido entendida tradicionalmente, es más que nada una ilusión producto de la inmersión del hombre en la cultura y el lenguaje. Es decir, aunque la conciencia se nos presenta como un sistema de procesamiento de información que funciona de manera lineal y está controlado por una entidad única -el Yo-, las neurociencias muestran que el cerebro en realidad es un sistema de procesamiento en paralelo que no cuenta con un sistema de control o un órgano en el que después se reúna toda la información para ser procesada (de nuevo) de forma lineal. Nuestro cerebro está constituido por una serie de módulos o circuitos más o menos independientes que funcionan a la vez tratando de resolver en ocasiones el mismo problema con herramientas distintas, pero sin ser capaces de comunicarse entre sí. El motivo de que en la conciencia esto se nos «presente» de un modo diferente es porque al sumergirnos en una cultura determinada, sobre la arquitectura o hardware cerebral de tipo paralelo el lenguaje implementa un software que es la conciencia. Este software es una «máquina virtual» que imita el funcionamiento de un sistema de procesamiento lineal. 18

15 Dennett, D. C.: La conciencia explicada: Una teoría interdisciplinar, Barcelona, Paidós, 1991/1995, pp. 423-441.

16 Una década después de la aparición de La conciencia explicada Dennett publicó otros dos artículos que vuelven a tratar el tema del Yo y de la responsabilidad, aunque sus aportaciones son mucho menores que la de las obras anteriores. En "In Darwin's Wake, Where Am I?" Dennett aborda el problema de la responsabilidad atendiendo sobre todo al pensamiento creativo (Proceedings and Addresses of the American Philosophical Association, 75, 2001, pp. 13-30). Por su parte en "The Self as a Responding-and Responsible-Artifact" explica cómo el Yo surge al tiempo con la conciencia por la necesidad social de dar cuenta de nuestras acciones y hacernos responsables de las mismas (Annals New York Academy of Science, 1001, 2003, pp. 39-50).

17 Dennett, D. C.: Dulces sueños: Obstáculos filosóficos para una ciencia de la conciencia, Buenos Aires, Katz, 2005/2006, p. 159.

18 Dennett, D.C.: La conciencia explicada, op. cit. p. 223. 
Es importante destacar que esta máquina virtual -que Dennett denomina joyceana en honor al escritor irlandés James Joyce y a su técnica del «flujo de conciencia»- no funciona realmente con un procesamiento lineal, sino que simplemente imita los inputs y outputs que tendría si siguiese un procesamiento lineal. En realidad el cerebro funciona de tal modo que existen al mismo tiempo infinidad de circuitos cerebrales elaborando de forma paralela posibles contenidos, fragmentos narrativos que compiten entre sí, de modo que sólo algunos de ellos tendrán efecto sobre otras actividades cerebrales, mientras que el resto desaparecerán sin dejar huella. Pero a pesar de nuestros informes introspectivos, los contenidos «ganadores» no son aquellos elegidos por un Yo que funcione a modo de «discriminador central». Cuando producimos un acto de habla -ya sea en voz alta o para nosotros mismos- aquellos contenidos narrativos que tienen mayor fuerza o son más «populares» en nuestro cerebro en ese momento se imponen sobre el resto y toman el control de nuestros labios. ${ }^{19}$ En este sentido aquello de lo que diríamos ser conscientes con respecto a un tema concreto cambia constantemente. No somos conscientes de algo y entonces lo decimos, sino que en cierto sentido nos hacemos conscientes al decirlo, ya que aquello que se encuentra en la conciencia en un determinado momento es simplemente un intento de fijar un contenido del continuo flujo de cambios que se están produciendo en todo momento en distintos lugares del cerebro. ${ }^{20}$ Los famosos lapsus lingüísticos serían una muestra de que ésta es en realidad la forma en que funciona el cerebro. Cuando «queremos» decir una palabra, pero a la hora de expresarnos resulta que decimos otra, o una mezcla de ambas, no es cierto que «en realidad» quisiéramos decir una cosa o la otra. Lo que ha sucedido es que el cerebro elaboraba ambas opciones al mismo tiempo en circuitos paralelos -además de seguramente varias decenas de opciones más-, pero en el momento de realizar el acto de habla ninguna de ellas tenía una fuerza mayor que la otra. En términos de la última revisión de la teoría, las dos opciones eran las más «populares» en el cerebro, pero ninguna de las dos lo era lo suficiente como para tomar el control absoluto, por lo que ambas intervinieron en la ejecución del discurso provocando que el resultado final fuese una mezcla incoherente de las dos.

19 Dennett profundiza en esta idea en "How to do Other Things with Words" (Royal Institute of Philosophy Supplement, 42, 1997, p. 233).

20 En consecuencia, el investigador que interrogue a un sujeto para saber de qué es consciente éste deberá tener en cuenta que el momento en el que decida hacer la pregunta estará influyendo en la respuesta del sujeto, pues estará interrumpiendo la competición entre los distintos discursos posibles dando como ganador al mejor situado en ese momento (Dennett, D.C.: La conciencia explicada, op. cit., pp. 148-150). Esto por supuesto no significa que ese discurso sea el contenido «final»o «definitivo» de la conciencia, pues seguirá siendo modificado en la memoria, como mostraré un poco más adelante. Pero todavía más chocante es que esto tampoco implica que hubiese un contenido «primero» o «original» de la conciencia a partir del cual se haya ido degradando la información. Siempre hay multitud de fragmentos narrativos en distintos espacios y tiempos en el cerebro siendo procesados a la vez, y la pregunta por el contenido «original» en la conciencia carece de sentido. 
Un último aspecto importante sobre la teoría de la conciencia de Dennett es la relación que se da entre ésta y la memoria, que permite entender mejor cómo funciona la máquina joyceana dentro del modelo cerebral de Versiones Múltiples. En la primera versión de su teoría señalaba Dennett que «el modelo de las Versiones Múltiples convierte al acto de "escribir en la memoria" en el criterio mismo para la conciencia» ${ }^{21}$. Afirmación que cobra aún más fuerza en la revisión del modelo, cuando llega a decir que la memoria episódica -nuestra capacidad de recordar en cualquier momento eventos particulares de nuestra propia vida- «es la característica más importante de la conciencia y, de hecho, lo más cercano al rasgo distintivo de la conciencia que podamos encontrar» 22 . Esto podría hacernos pensar que el contenido de la conciencia, aunque variable en un principio, queda fijado una vez es expresado verbalmente y almacenado en la memoria. Sin embargo aquí entra en funcionamiento la máquina joyceana, que es entendida como un hábito de autoestimulación adquirido culturalmente. Los científicos que trabajan sobre el aprendizaje animal señalan que según la inteligencia de la especie con la que se trabaje son necesarias cuatro, cinco o cincuenta repeticiones del mismo experimento para que el animal aprenda el contenido que se le quiere enseñar. Sin embargo, aunque aprendan el contenido, es dudoso que los animales recuerden alguno de los eventos particulares de los experimentos. Los animales, según la posición defendida por Dennett, no serían capaces de recordar a largo plazo eventos concretos de su propia vida debido a que no pueden experimentarla las veces suficientes como para hacerlo. El hombre sin embargo es capaz de recordar algunos eventos aislados de su vida a partir de una única repetición. Esto no se debe únicamente a que la memoria humana sea más hábil que la de otros animales de forma natural, sino también a que la máquina joyceana, trabajando a partir de la versión más cerebralmente popular de un evento, nos posibilita repetirnos a nosotros mismos una reconstrucción verbal de dicho evento cuantas veces queramos, ayudándonos a formar así un recuerdo perdurable. De este modo, el recuerdo de un evento no es tal, sino que en realidad es el recuerdo de una expresión lingüística. ${ }^{23} \mathrm{Al}$ mismo tiempo, y en consecuencia, los recuerdos así formados no son estables, ya que cada vez que se reconstruyen verbalizándolos de nuevo se les añaden variantes lingüísticas que adornan, amplían o reducen determinados aspectos. De este modo las historias que contamos sobre nosotros mismos se componen de recuerdos que ni son los únicos que podríamos haber tenido -ya que la versión más popular en nuestro cerebro que dio origen a cada uno de nuestros recuerdos podría haber sido otra-, ni son estables -ya que cada vez que los recordamos los reconstruimos de nuevo-.

21 Dennett, D.C.: La conciencia explicada, op. cit., p. 145.

22 Dennett, D.C.: Dulces sueños, op. cit., p. 196.

23 Ibid., p. 195. 
En este contexto resulta mucho más comprensible la teoría del Yo como centro de gravedad narrativa. Para Dennett el Yo no es una entidad real que deba buscarse en el cerebro o identificarse con alguna otra entidad física. Ni siquiera con nuestro cuerpo en su totalidad. El Yo es una abstracción fruto de la interpretación de nuestro discurso. Dado que todos los enunciados que proferimos en primera persona tiene por sujeto a un «yo», se crea la ilusión de que hay un autor del texto, un ente unificado que ha decidido producir el discurso. Sin embargo no es así, ya que como acabamos de ver el discurso es simplemente la verbalización de aquellos fragmentos narrativos producidos de forma independiente en distintos lugares del cerebro que estaban en mejor disposición para producir el habla. No hay un Yo que tome decisiones en nuestro interior, sino que para interpretar y estructurar toda la información que tiene sobre nosotros, nuestro cerebro la organiza en torno a un punto de representación. Este punto es una ficción teórica que Dennett llama «centro de gravedad narrativa» por analogía con el centro de gravedad de los objetos físicos. Pues al igual que el centro de gravedad sirve a los físicos para predecir e interpretar el comportamiento de las mesas, las sillas o las lámparas pero no se puede identificar con ningún componente físico de dichos objetos, el centro de gravedad narrativa nos sirve a los humanos para predecir e interpretar tanto nuestro propio comportamiento como el de nuestros semejantes a pesar de no ser un objeto que exista en el mundo real aparte de los átomos que forman nuestro cuerpo.

El papel central que tiene el Yo en nuestra configuración se evidencia en la idea de Dennett de que existe una especie de instinto biológico en el ser humano que le mueve a contar una historia acerca de sí mismo como mecanismo de defensa. Al igual que las arañas están condicionadas para construir telarañas de forma natural, los seres humanos tendrían la necesidad de contar su historia, siendo así inevitable la formación del Yo, que formaría parte de su fenotipo ampliado. ${ }^{24}$ Sin embargo no se puede olvidar que, pese a las apariencias, en realidad no es un Yo el que cuenta la historia. La historia es producto de la actividad del cerebro, y el Yo es producto de la historia. Son nuestras historias las que nos crean a nosotros y no al revés. ${ }^{25} \mathrm{En}$ este sentido sería posible que la historia que «cuenta» nuestro cerebro involucrara no a un Yo, sino a varios, como demuestran los casos de personalidad múltiple. La explicación sin embargo no sería que en un mismo cuerpo conviven varias entidades fantasmales o Yoes que compiten por el control corporal. Simplemente sucedería que ante la dificultad de interpretar su propia historia postulando un único centro de gravedad narrativa, su cerebro habría decidido organizar su información en torno a varios centros de gravedad narrativa, logrando así un mayor poder explicativo y una mayor coherencia.

\footnotetext{
24 Dennett, D. C.: "The Origins of Selves", op. cit., pp. 167-168.

25 Dennett, D. C.: La conciencia explicada, op. cit., p. 428.
} 
De este modo nuestra existencia es en cierto sentido análoga a la de los personajes de una novela. Sólo somos un punto en el discurso. Somos una abstracción útil que sirve para simplificar nuestra forma de comprender el mundo y a nosotros mismos. La principal diferencia entre nuestros Yoes y los personajes literarios es que éstos últimos se presentan una vez que su escritura ha finalizado, de modo que lo que en ellos es indeterminado - ¿qué más pensó Raskolnikov mientras estaba sentado en la estación de policía?- permanecerá así para siempre. Por el contrario los Yoes se encuentran siempre en proceso de escritura, por lo que cualquier aspecto indeterminado - ¿me gusta realmente esa mujer?- puede volverse determinado más adelante. Esto a su vez parece indicar que para los Yoes, al contrario que para los personajes literarios, sí rige el principio de bivalencia. Es decir, que mientras que no es ni cierto ni falso, por ejemplo, que Sherlock Holmes tuviese un lunar en el hombro izquierdo dado que no hay ninguna frase en las obras de Arthur Conan Doyle que nos permita dar una respuesta, con respecto a un personaje histórico como Aristóteles la respuesta tiene que ser afirmativa o negativa, a pesar de que no haya forma de que nosotros podamos averiguarlo. Pero volveré sobre esto en el próximo apartado.

Lo dicho hasta ahora se puede considerar como la suma de los motivos por los que Dennett suscribe la tesis psicológica de la Narratividad, que es el requisito mínimo que todas las teorías del Yo narrativo tienen en común. Más sutiles debemos ser sin embargo para ver el apoyo de la teoría de Dennett a la tesis ética de la Narratividad, que señala que comprender la propia vida en forma narrativa es algo necesario para tener una vida buena o para desarrollarse plenamente como persona. Señalé al principio de estas páginas que Dennett no se acerca de un modo tan explícito al tema de la ética, pero sin embargo sí lo hace tangencialmente al hablar del libre albedrío en La libertad de acción. Por otro lado, en La conciencia explicada también hace alusión al tema estrella del debate sobre la identidad personal entre los partidarios del enfoque objetivo, la supervivencia, que en última instancia también se relaciona con las otras tres características señaladas por Schechtman como vinculadas al problema de la identidad personal: la preocupación prudencial por nuestro bienestar, la compensación futura y la responsabilidad moral. Vayamos por partes.

Según Strawson, la idea que late de fondo en la tesis ética de la Narratividad es que es necesario tener una comprensión profunda de uno mismo para poder actuar de una forma ética, y que esta auto-comprensión sólo puede darse de forma Narrativa. ${ }^{26}$ En este sentido podemos ver que para Dennett la auto-reflexión nos lleva a un mayor auto-conocimiento, de tal modo que reflexionar sobre nosotros mismos nos hace más maduros y responsables. ${ }^{27}$ Sin embargo esta preocupación

\footnotetext{
26 Strawson, G.: Contra la Narratividad, op. cit.

27 Dennett, D. C.: La libertad de acción, op. cit., pp. 105-106.
} 
por la responsabilidad nos impulsa a preocuparnos en mayor medida por la diferencia entre aquellas acciones que realmente nos son imputables -dado que elegimos hacerlas libremente- y aquellas otras que no pudimos evitar, que nos acaecieron, y de las que por tanto no somos responsables. Pues aparentemente, por poner un ejemplo, aunque no se nos puede responsabilizar del hecho de que una situación nos haya provocado ira, sí que somos responsables de controlar las acciones que la ira nos invita a hacer. De este modo la preocupación por la responsabilidad moral nos hace profundizar en la ilusión del Yo como responsable último de nuestras decisiones, influyendo de modo decisivo en su formación. ${ }^{28}$ Esto lleva a Dennett a concluir que cuando nacemos no tenemos un Yo desarrollado y que sólo mediante ciertos procesos de aprendizaje y de maduración - la cual se alcanza reflexionando narrativamente sobre nosotros mismos- llegamos a convertirnos en adultos morales autoconscientes. ${ }^{29}$ Lo cual se parece mucho a la formulación de Strawson de que tener una comprensión narrativa de uno mismo es necesario para desarrollarse plenamente como persona.

Frente a la previsible acusación de cómo puede un Yo que no es real asumir responsabilidades, Dennett empieza a aventurarse en lo que será el mayor problema de su teoría. Pues aunque no somos más que una creación ficticia de algún modo existimos realmente, hasta el punto de que como centros de gravedad narrativa lo que nos debe preocupar en nuestra supervivencia no es la supervivencia de nuestro cuerpo, sino la de la narración que nos crea. Así dice Dennett que aunque es necesario que exista nuestro cuerpo para darnos vida, podríamos sobrevivir a su muerte si por ejemplo se «trasvasase» nuestra información cerebral a un ordenador. Sin embargo Dennett no cae en la cuenta de que, siguiendo con su línea de pensamiento, lo que es importante para la supervivencia del Yo no es «el programa que corre en el ordenador de su cerebro» 30 , como llega a decir, ya que no somos ese programa. Según su propia postura, lo que somos es una abstracción teórica, un punto en el tejido de discursos que nos forma. Pues no estamos constituidos sólo por nuestro discurso, sino también por el de nuestra familia, nuestros amigos, nuestros conocidos... ${ }^{31}$ Dado que la conciencia surge en un medio social como una herramienta para comprender y modificar los comportamientos de los otros, el Yo también posee esta característica, siendo su existencia fruto de una negociación entre nuestra narración y la de los que nos rodean. ${ }^{32}$ Siendo así, para ser coherente Dennett tendría que decir que lo importante en nuestra supervivencia en realidad no es nuestra actividad cerebral. Lo único que nos hace sobrevivir como Yoes es que siga existiendo la cre-

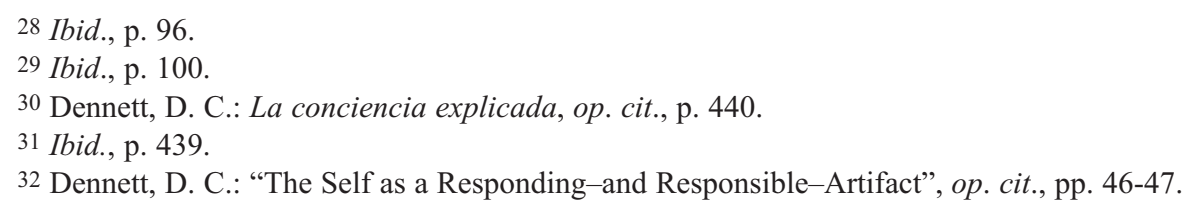


encia de que existimos y se sigan produciendo discursos en los que nosotros intervenimos. De este modo, mientras nuestra sociedad conserve la creencia de que el Yo desaparece con la muerte del cuerpo, moriremos con nuestro cuerpo aunque trasvasen nuestra información cerebral a un ordenador, pues todos los discursos dirán que hemos muerto y dejarán de hablar de nosotros. Y si viviésemos en una sociedad que estuviese de acuerdo en que el Yo sobreviviría en una estatua que estuviese cerca de nuestro cuerpo cuando exhalásemos nuestro último aliento, sobreviviríamos en esa estatua, aunque no hubiese ninguna sustancia ni información que pasase de un lugar a otro, ni la estatua fuese un organismo, no ya vivo, sino ni siquiera capaz de acción. ${ }^{33}$ En cualquier caso analizaremos los problemas de la teoría de Dennett en el próximo apartado.

\section{Críticas a los planteamientos narrativos}

Como señalaba al principio del artículo, las teorías narrativas han recibido importantes críticas en los últimos años. Algunas de ellas son específicas del planteamiento de Dennett, aunque la mayoría son extensibles al planteamiento narrativo en general como veremos a continuación.

Empezando por las primeras, la teoría de Dennett tiene un problema de carácter ontológico que parte de su confusa consideración del estatus de los elementos de ficción. Así, al mismo tiempo que nos asegura que los personajes de ficción no presentan problema alguno -dado que en realidad no existen-34 y que los Yoes sólo son otra ficción, también nos dice que se trata de dos tipos de ficciones diferentes, ${ }^{35}$ dado que los Yoes, como veíamos al final del apartado anterior, en cierto sentido sí existen. Para ellos, al contrario que para los personajes ficticios, parece regir el principio de bivalencia. De este modo da a entender que hay grados entre los elementos que no existen, de forma que unos son más inexistentes que otros, lo cual pare-

\footnotetext{
33 Es importante señalar que existen en la filosofía de Dennett elementos que permitirían negar este razonamiento. Así por ejemplo para Dennett algunas de las condiciones para ser una persona son ser racional, ser capaz de comportarse de determinado modo, ser capaz de comunicarse verbalmente y ser autoconsciente, condiciones que la estatua de mi ejemplo no cumpliría (Condiciones de la cualidad de persona, Cuadernos de crítica, 45, México: Universidad Nacional Autónoma de México, 1976/1989, pp. 8-11). En el mismo sentido defiende que en el caso de pacientes con el cerebro dividido no existen dos Yoes, pues un hemisferio cerebral es incapaz por sí solo de aglutinar bastante materia narrativa como para conformar un Yo, lo que también parece sugerir que es importante que exista un sujeto capaz de narrar en primera persona (La conciencia explicada, op. cit., p. 435). Sin embargo estas consideraciones deberían articularse con el hecho de que sólo somos un punto en el tejido de discursos formado tanto por nuestra narración como por las narraciones que otros hacen sobre nosotros, y eso es lo que pretendo poner de relieve con el ejemplo de la estatua.

34 Dennett, D. C.: La conciencia explicada, op. cit., p. 92.

35 Ibid., pp. 422-423.
} 
ce incoherente. ${ }^{36}$ En este sentido Velleman argumenta que pese a considerar que son ficticios, Dennett atribuye a los Yoes unas características y capacidades que sólo podrían tener si fuesen reales, por lo que concluye que el Yo dennettiano debe ser real. ${ }^{37}$ En cualquier caso, como veremos más adelante, la postura ontológica de Dennett es más complicada de lo que parece a primera vista, y podría solventar este problema con algunos pequeños ajustes, solucionando al mismo tiempo además otro problema ontológico que afecta a todas las teorías narrativas.

Un segundo problema específico de la teoría de Dennett reside en que no puede dar una explicación al hecho de que, pese a ser ficticios, los Yoes tienen como referencia el mundo real a la hora de construir sus relatos. ${ }^{38}$ Parece cierto que todos intentamos ser más o menos fieles a la realidad a la hora de contar nuestras vidas -a pesar de la escasa fiabilidad de nuestra memoria- y mientras que la «vida» de un personaje literario puede transformarse radicalmente en virtud de hechos imaginarios, no parece que haya ninguna circunstancia posible por la que yo mañana pueda convertirme por ejemplo en Marilyn Monroe -ni en una estatua, dicho sea de paso-. Aunque un golpe en la cabeza me hiciera adoptar los recuerdos y la personalidad que Marilyn tenía justo antes de morir de tal modo que fuese capaz de crear una narración en la que explicase cómo me convertí en Marilyn, nadie consideraría que ahora soy ella. Simplemente me habría vuelto loco. Una teoría narrativa parecida a la de Dennett que evita este problema es la de Schechtman, que postula lo que ella denomina la «restricción de la realidad», que demanda que para poder constituir nuestra identidad nuestras narraciones deben conformarse al menos hasta cierto punto a los hechos reales en los que estuvieron involucrados nuestros cuerpos. ${ }^{39}$ Otra solución de corte más dennettiano sería hacer más hincapié en el hecho de que nuestros Yoes no están constituidos únicamente por nuestras propias narraciones, sino también por las narraciones de otros, que podrían servir de control. Pero también volveré sobre esto antes de terminar.

Otro problema de la teoría de Dennett pero que afecta igualmente al resto de teorías narrativas es que utilizan el término «narración» de forma ambigua, no estando claro en muchos casos ni qué consideran que es una narración ni qué acceso consciente se supone que tienen las personas a sus propias narraciones, pues es evidente que raras veces una persona cuenta una narración explícita de su vida al completo. ${ }^{40}$ En este sentido los narrativistas utilizan demostraciones de que comprendemos el mundo de forma narrativa en un sentido mínimo o trivial para posteriormente sacar conclusiones sobre las personas que implican un sentido de narra-

\footnotetext{
36 Carr, D.: "Phenomenology and Fiction in Dennett", op. cit., p. 339.

37 Velleman, J. D.: "The Self as Narrator", op. cit.

38 McCarthy, J.: Dennett and Ricoeur on the Narrative Self, op. cit., pp. 86-88.

39 Schechtman, M.: The Constitution of Selves, op. cit., pp. 119-120.

$40 \mathrm{Vid}$. Lamarque, P.: "On Not Expecting Too Much from Narrative”, op. cit., pp. 402-406.
} 
ción mucho más fuerte. En el caso de Dennett, únicamente demuestra que la conciencia es un producto del lenguaje que aparentemente procesa la información de modo lineal, pero no explica qué es lo que le permite dar el salto desde aquí a la afirmación de que además es narrativa. Si lo que quiere decir es que toda construcción lingüística que presente información de forma lineal es una narración, sin duda está utilizando el término en un sentido cuanto menos trivial. Sin embargo esto no le impide hacer comparaciones entre estos Yoes trivialmente narrativos y los personajes literarios, que son narrativos en un sentido mucho más fuerte que el de ser una construcción lingüística. Para tener un carácter narrativo como el que tienen los personajes literarios nuestra conciencia debería procesar la información no simplemente de una forma lineal, sino además hacerlo según la lógica y la estructura de las obras literarias, cosa que el modelo de Versiones Múltiples de Dennett no implica. De este modo, las comparaciones que hace entre personas y personajes, que le permiten acentuar el carácter ficticio de las primeras, se basan en un uso ambiguo del concepto de narración por lo que las conclusiones que extrae de ellas no están justificadas.

Un último problema de la teoría de Dennett que también afecta al resto de teorías narrativas es que no da cuenta de los aspectos fenoménicos del Yo. Por un lado, Dennett parece confundir lo que es una historia a un nivel fáctico con su interpretación. Si volvemos al ejemplo de Sherlock Holmes, a nivel fáctico sus historias están compuestas por los libros escritos por Conan Doyle, del mismo modo que nuestras historias están compuestas por nuestras expresiones orales sobre nosotros mismos. Sin embargo las manchas de tinta sobre un papel o las ondas sonoras que emiten nuestras bocas no son historias por sí mismas. Algo no es una historia hasta que es interpretado como tal por alguien. Las obras sobre Sherlock Holmes necesitan de un lector consciente para ser historias, y nuestros Yoes narrativos necesitan de un Yo consciente que interprete nuestras narraciones. ${ }^{41}$ En la misma línea de argumentación podemos ver que existe un sentido fenomenológico del Yo previo a toda narración. Un sentido que nos permite sentir, por ejemplo, un dolor como propio antes de situarlo en una narración de ningún tipo. Pensemos en un enfermo de Alzheimer que no tenga ninguna capacidad para articular narrativamente su vida. A pesar de este hecho, no tenemos ningún motivo para suponer que cuando siente un dolor lo siente como un dolor ajeno o impersonal en vez de como un dolor que le está sucediendo a él. Siendo así, necesitamos postular la existencia de un Yo fenomenológico que no depende de nuestras narraciones. 42 Un Yo fenomenológico que bien podría ser el intérprete de nuestras historias.

Si vamos todavía un poco más lejos en esta misma dirección, podemos pensar en las críticas que hace Strawson al enfoque narrativo. ${ }^{43}$ Para Strawson la tesis psi-

41 Carr, D.: "Phenomenology and Fiction in Dennett", op. cit., pp. 341-342.

42 Zahavi, D.: "Self and Other: The Limits of Narrative Understanding", op. cit., pp. 192-193.

43 Strawson, G.: Contra la Narratividad, op. cit. 
cológica de la Narratividad es falsa pues, poniéndose a sí mismo como ejemplo, señala que existen seres humanos cuya experiencia fenomenológica del Yo es esencialmente Episódica, es decir, no tienen ninguna sensación de tener una existencia temporal dilatada y por tanto ningún impulso hacia la comprensión narrativa de sus vidas. Por su parte, añade Strawson, la tesis ética de la Narratividad además de falsa es potencialmente perniciosa para este tipo de sujetos. Pues no sólo no necesitan comprender su vida en términos narrativos para poder desarrollar un comportamiento ético, sino que hacerlo les resulta costoso hasta el punto de poder causarles graves trastornos psicológicos. Poniendo en relación esta crítica con la anterior podemos ver que no sólo existe un sentido fenomenológico del Yo que es previo a toda narración, sino que existen personas que no sienten la necesidad de construir a partir de éste un Yo narrativo más amplio.

\section{El nuevo planteamiento narrativo y la aportación de Dennett al mismo}

Tratando de responder a Strawson, recientemente Schechtman sugirió que el problema de las teorías narrativas podría ser que hasta la fecha habían estado ignorando una tensión subyacente entre dos concepciones distintas de lo que somos. Para resolverla propuso distinguir entre los términos «Yo»y «persona» como si se refiriesen a dos entidades diferentes, la primera de naturaleza fenomenológica y la segunda de naturaleza social. ${ }^{44}$ De este modo tendríamos por un lado al Yo, que no tendría por qué tener una duración temporal extensa, que sería trivialmente narrativo y que daría cuenta de la dimensión fenomenológica de nuestra existencia; y por otro lado a la persona, cuya duración se extendería a lo largo de prácticamente toda la vida del ser humano, cuya configuración sería narrativa en un sentido más fuerte y que daría cuenta de nuestra existencia social y por extensión de las cuatro características de la identidad personal: la supervivencia, la preocupación prudencial por nuestro bienestar, la compensación futura y la responsabilidad moral.

La propuesta de Schechtman de distinguir entre Yoes y personas ha sido bien acogida por prácticamente todos los participantes en el debate contemporáneo sobre el Yo narrativo. ${ }^{45}$ Sin embargo todavía deja muchas dudas en el aire en lo atinente al problema de la identidad personal. Principalmente porque las dos tesis de la Narratividad tienen un profundo carácter psicológico y no queda claro cómo se pueden aplicar a unas entidades de marcado carácter social como las personas. Por otro

\footnotetext{
44 Schechtman, M.: "Stories, Lives and Basic Survival: A Refinement and Defense of the Narrative View", en D. D. Hutto (ed.), Narrative and Understanding Persons, op. cit., pp. 169-170.

45 Vid. Menary, R.: "Embodied Narratives", Journal of Consciousness Studies, 15, 2008, pp. 63-84; Schechtman, M.: "The Narrative Self", en S. Gallagher (ed.), The Oxford Handbook of the Self, Oxford, Oxford University Press, 2011, pp. 394-416; Zahavi, D.: "Self and Other: The Limits of Narrative Understanding”, op. cit., pp. 179-201.
} 
lado tampoco queda claro qué marco ontológico nos puede permitir distinguir entre tres entidades pretendidamente reales y relativamente independientes entre sí -Yoes, personas y seres humanos- que ocupan un mismo espacio físico. Es para aclarar estos dos asuntos para los que considero que la teoría de Dennett podría ser de utilidad.

En cuanto al primer problema, la teoría de Dennett podría ayudar a reformular la tesis ética de la Narratividad haciéndonos recordar que en su origen lo que se encuentra es la necesidad de dar cabida a la dimensión social de nuestra existencia. Lo importante para los teóricos de la identidad narrativa era contar con un criterio de identidad lo bastante fuerte como para servir de base a los problemas presentados por las cuatro características, especialmente la responsabilidad moral, y no el postular la necesidad de que las personas tuviesen una auto-comprensión narrativa de sus vidas en un sentido fuerte para poder actuar de forma ética. Esto era un medio para alcanzar un fin, no un fin en sí mismo. Siendo así, si acentuamos la tesis de Dennett de que nuestra identidad no está constituida únicamente por nuestros relatos, sino también por los relatos de los que nos rodean podríamos reformular la tesis ética de la Narratividad convirtiéndola en una tesis social de la Narratividad que afirmase que las personas deben ser capaces de aceptar ciertas restricciones y demandas hechas por otras personas, de tal modo que su identidad se construya mediante la negociación entre el relato propio y los relatos de los otros.

Con respecto al segundo problema, creo que la postura ontológica de Dennett, ampliamente incomprendida, está en mejor situación que otras para afrontarlo. Para Dennett el mejor modo de comprender la conciencia es abandonando la descripción física del mundo, pues lo físico es incapaz de explicar lo fenoménico. En su lugar propone que la conciencia es un fenómeno real del mundo pero que sólo es descriptible desde la actitud intencional. La actitud intencional es la que se obtiene cuando a un objeto del mundo se le considera un agente racional con creencias, deseos e intenciones. ${ }^{46}$ De este modo según Dennett tenemos distintas actitudes interpretativas con las que podemos acercarnos al mundo -Dennett define tres, pero señala que existen innumerables-, ninguna de las cuales es más cierta que las otras, pues todas se basan en la detección de patrones reales de comportamiento: patrones físicos en el caso de la actitud física, patrones biológicos en el caso de la actitud de diseño y patrones mentales en el caso de la actitud intencional. Esta postura, sin duda demasiado compleja para explicarla aquí en profundidad, ${ }^{47}$ tiene el problema de estar a medio camino entre el realismo y el instrumentalismo. Sobre todo porque parece ser más realista que instrumentalista acerca de las actitudes física y de diseño pero más instrumentalista que realista con respecto a la intencional, lo que parece acercarle al

46 Dennett, D. C.: La actitud intencional, Barcelona, Gedisa, 1987/1998, pp. 28-29.

47 Vid. Dennett, D. C.: "Pautas reales", Agora, 11/2, 1991/1992, pp. 97-119. 
eliminativismo. ${ }^{48}$ No es mi intención abordar este problema de otra teoría de Dennett en este momento, pero sin embargo sí creo importante señalar que un cierto trabajo de corrección sobre ella podría otorgarnos un marco ontológico prometedor con el que abordar el problema de la identidad personal. Abandonando en primer lugar la tendencia a favorecer la actitud física frente a otras y reconociendo que los objetos físicos son tan reales o ficticios como los descritos desde otras actitudes como la actitud intencional, desde este marco podríamos definir tres actitudes interpretativas con respecto a la realidad -la actitud de diseño, la actitud intencional y la actitud social, por ejemplo- que detectasen patrones reales de comportamiento de distinto tipo -biológicos, mentales y sociales-, de tal modo que según qué actitud interpretativa estemos asumiendo seremos seres humanos, Yoes o personas, respectivamente. Las dos primeras actitudes ya han sido definidas por Dennett, por lo que no sería necesario aportar mucho material para llevar a cabo este trabajo. La actitud social sin embargo no tiene precedente en la obra del filósofo americano y su definición debería ser abordada con un espíritu más emprendedor. En cualquier caso este proyecto es mucho más ambicioso de lo que estas páginas nos permiten, por lo que tendrá que desarrollarse en otro lugar. ${ }^{49}$

\section{Referencias bibliográficas}

BRuner, J. S.: "Life as Narrative", Social Research, 54, 1987, pp. 11-32.

Butler, J.: "Of Personal Identity", en J. Perry (ed.), Personal Identity, Berkeley and Los Angeles, University of California Press, 2008, pp. 99-106.

CARR, D.: "Phenomenology and Fiction in Dennett", International Journal of Philosophical Studies, 6, 1998, pp. 331-344.

Christman, J.: "Narrative Unity as Condition of Personhood", Metaphilosophy, 35, 2004, pp. 695-713.

Dennett, D. C.: Condiciones de la cualidad de persona, Cuadernos de crítica, 45, México: Universidad Nacional Autónoma de México, 1976/1989.

Dennett, D. C.: "Reflexiones", en D. R. Hofstadter y D. C. Dennett (eds.), El ojo de la mente: Fantasías y reflexiones sobre el yo y el alma, Buenos Aires, Editorial Sudamericana, 1981/1983, pp. 348-352.

DenNetT, D. C.: La libertad de acción: Un análisis de la exigencia de libre albedrio, Barcelona, Gedisa, 1984/2000.

\footnotetext{
48 En este sentido es un claro ejemplo de este problema su afirmación de que el Yo «no es uno de los objetos reales del universo que exista además de los átomos», dando a entender así que los átomos, a diferencia de los Yoes, si son reales (Dennett, D. C.: "El Yo como centro de gravedad narrativa", op. cit.).

49 Este tema es precisamente uno de los aspectos centrales de la tesis doctoral del autor de este artículo.
} 
DennetT, D. C.: La actitud intencional, Barcelona, Gedisa, 1987/1998.

DennetT, D. C.: "El Yo como centro de gravedad narrativa", Logos: Anales del Seminario de Metafísica, 1988/2013, vol. 46.

Dennett, D. C.: 'The Origins of Selves', Cogito, 3, 1989, pp. 163-173.

DenNetT, D. C.: La conciencia explicada: Una teoría interdisciplinar, Barcelona, Paidós, 1991/1995.

DennetT, D. C.: "Pautas reales”, Agora, 11/2, 1991/1992, pp. 97-119.

DennetT, D. C. "How to Do Other Things with Words", Royal Institute of Philosophy Supplement, 42, 1997, pp. 219-235.

Dennett, D. C.: "In Darwin's Wake, Where Am I?", Proceedings and Addresses of the American Philosophical Association, 75, 2001, pp. 13-30.

DennetT, D. C.: "The Self as a Responding-and Responsible-Artifact", Annals New York Academy of Science, 1001, 2003, pp. 39-50.

DennetT, D. C.: Dulces sueños: Obstáculos filosóficos para una ciencia de la conciencia, Buenos Aires, Katz, 2005/2006.

Lamarque, P.: “On Not Expecting Too Much From Narrative”, Mind \& Language, 19, 2004, pp. 393-408.

Lamarque, P.: "On the Distance between Literary Narratives and Real-Life Narratives", en en D. D. Hutto (ed.), Narrative and Understanding Persons, Cambridge, Cambridge University Press, 2007, pp. 117-132.

Lewis, D. K.: Supervivencia e identidad, Cuadernos de crítica, 27, México, Universidad Nacional Autónoma de México, 1976/1984.

Locke, J.: Ensayo sobre el entendimiento humano, México, Fondo de Cultura Económica, 1690/1999.

MacIntyre, A.: Tras la virtud, Barcelona, Crítica, 1981/2004.

McCARthy, J.: Dennett and Ricoeur on the Narrative Self, Amherst, NY, Humanity Books, 2007.

MEnARY, R.: “Embodied Narratives”, Journal of Consciousness Studies, 15, 2008, pp. 63-84.

PARFIT, D.: Identidad personal, Cuadernos de crítica, 25, México, Universidad Nacional Autónoma de México, 1971/1983.

PARFIT, D.: Razones y personas, Boadilla del Monte (Madrid), A. Machado Libros, 1984/2004.

Perry, J.: "Can the Self Divide?", The Journal of Philosophy, 69, 1972, pp. 463488.

REID, T.: “Of Mr. Locke's Account of Our Personal Identity”, en J. Perry (ed.), Personal Identity, Berkeley and Los Angeles, University of California Press, 2008, pp. 113-118.

Ricoeur, P.: Sí mismo como otro, Madrid, Siglo XXI, 1990/1996.

Schechtman, M.: The Constitution of Selves, Ithaca, NY, Cornell University Press, 1996. 
Schechtman, M.: "Stories, Lives and Basic Survival: A Refinement and Defense of the Narrative View", en D. D. Hutto (ed.), Narrative and Understanding Persons, Cambridge, Cambridge University Press, 2007, pp. 155-178.

Schechtman, M.: "The Narrative Self", en S. Gallagher (ed.), The Oxford Handbook of the Self, Oxford, Oxford University Press, 2011, pp. 394-416.

Shoemaker, S.: "Personal Identity and Memory", The Journal of Philosophy, 56, 1959, pp. 868-882.

Slors, M.: "Two Conceptions of Psychological Continuity", Philosophical Explorations, 1, 1998, pp. 61-80.

Strawson, G.: Contra la Narratividad, Cuadernos de crítica, México, Universidad Nacional Autónoma de México, 2004/en prensa.

TAYLOR, C.: Fuentes del yo: La construcción de la identidad moderna, Barcelona, Paidós, 1996.

Velleman, J. D.: "The Self as Narrator", en Self to Self: Selected Essays, Cambridge, Cambridge University Press, 2006, pp. 203-223.

VICE, S.: "Literature and the Narrative Self", Philosophy, 78, 2003, pp. 93-108.

Williams, B.: "Personal Identity and Individuation", Proceedings of the Aristotelian Society, 57, 1957, pp. 229-252.

ZaHAVI, D.: "Self and Other: The Limits of Narrative Understanding", en D. D. Hutto (ed.), Narrative and Understanding Persons, Cambridge, Cambridge University Press, 2007, pp. 179-201.

Alfonso Muñoz Corcuera

Departamento de Teoría del Conocimiento,

Estética e Historia del Pensamiento

Facultad de Filosofía

Universidad Complutense de Madrid

alfonso.m.corcuera@gmail.com 